\title{
Intervenções para a prevenção de condições crônicas: avaliação da prevalência de hemoglobinas variantes em população estudantil - Alfenas, MG
}

\author{
Ramon Alves de Oliveira PAULA ${ }^{1}$ \\ Guilherme Silveira DUARTE ${ }^{2}$ \\ Bruna Costa de França MARQUES \\ Alessandra dos Santos Danziger SILVÉRIO ${ }^{4}$ \\ Maria Rita RODRIGUES 5 \\ Fernanda Borges de Araujo PAULA 6 \\ Stella Maris da Silveira DUARTE ${ }^{7}$
}

\begin{abstract}
${ }^{1}$ Graduando em Farmácia pela UNIFAL-MG. E-mail: ramon-alves@ hotmail.com
${ }^{2}$ Graduando em Medicina pela UNIFENAS.

${ }^{3}$ Graduanda em Biomedicina pela UNIFAL-MG.

${ }^{4}$ Farmacêutica, Professora da UNIFENAS.

${ }^{5}$ Farmacêutica, Professora Adjunta. UNIFAL-MG, Departamento de Análises Clínicas e Toxicológicas.

${ }^{6}$ Farmacêutica, Professora Associada. UNIFAL-MG, Departamento de Análises Clínicas e Toxicológicas.

${ }^{7}$ Farmacêutica, Professora Associada. UNIFAL-MG, Departamento de Análises Clínicas e Toxicológicas.
\end{abstract}

Recebido em: 12/02/2013 - Aprovado em: 12/07/2013 - Disponibilizado em: 15/08/2013

\section{RESUMO}

As hemoglobinopatias são doenças hereditárias, de comum ocorrência na sociedade. Como exemplo, tem-se as hemoglobinas variantes, das quais a hemoglobina $\mathrm{S}$ e hemoglobina $\mathrm{C}$ são consideradas as de maior incidência na população. O presente estudo compreende a análise de uma população de estudantes da cidade de Alfenas, Minas Gerais, com relação à presença das hemoglobinas variantes $\mathrm{HbS}$ e $\mathrm{HbC}$, seja em homozigose ou heterozigose. Este trabalho contou com a participação de indivíduos voluntários de ambos os sexos e variadas etnias, de idade igual ou superior a 18 anos, com a aplicação do termo de consentimento livre e esclarecido. Foram analisadas 600 amostras sanguíneas, sendo que 96,50\% apresentaram resultados normais. Por outro lado, 3,50\% da população estudada foram diagnosticados portadores de hemoglobinas variantes. Além disso, dentre os indivíduos normais, verificou-se que 0,83\% foram diagnosticados como heterozigotos para hemoglobina $\mathrm{C}$ e que 2,67\% são portadores do traço falcêmico. Dessa forma, este estudo alerta e firma a necessidade do diagnóstico antecipado das hemoglobinas variantes, além da garantia do acompanhamento médico adequado e do aconselhamento genético à população, conferindo maior segurança e qualidade de vida às gerações subsequentes.

PALAVRAS-CHAVE: Hemoglobinopatia. Hemoglobina Variante. Hemoglobina S. Hemoglobina C. Traço Falciforme.

\section{Interventions for the prevention of chronic conditions: evaluation of prevalence of hemoglobin variants in student population - Alfenas, MG}

\section{ABSTRACT}

Hemoglobinopathies are hereditary diseases of common occurrence in society. As an example, there are the hemoglobin variants, in which the hemoglobin $\mathrm{S}$ and hemoglobin $\mathrm{C}$ are considered the highest incidence in the population. This study comprises the analysis of a student population of the city of Alfenas, Minas Gerais, considering the presence of hemoglobin variants $\mathrm{HbS}$ and $\mathrm{HbC}$, either homozygous or heterozygous. This work included the participation of volunteers of both sexes and varied ethnicities, with age equal or under 18 years, with the application of 
the term of consent. Were analyzed 600 blood samples, of which $96.50 \%$ had normal results. On the other hand, $3.50 \%$ of the analyzed population were diagnosed like carriers of hemoglobin variants. Furthermore, among normal individuals, it was found that $0.83 \%$ were diagnosed as heterozygous for hemoglobin $\mathrm{C}$ and $2.67 \%$ carry the sickle cell trait. Thus, this study emphasizes the need of the early diagnosis of hemoglobin variants, besides the assurance of adequate medical care and genetic counseling to the population, improving safety and quality of life for subsequent generations.

KEYWORDS: Hemoglobinopathy. Hemoglobin variant. Hemoglobin S. Hemoglobin C. Sickle Cell Trait.

\section{INTRODUÇÃO}

As hemoglobinopatias, também conhecidas como distúrbios hereditários da hemoglobina humana, são as doenças da fração proteica da hemoglobina, de caráter genético, mais comuns e afetam um grande número de indivíduos no mundo, com diversas complicações clínicas variando desde quase imperceptíveis até consequências letais (BONINI-DOMINGOS et al., 2004).

No Brasil, a ocorrência é muito frequente e influenciada pelos grupos étnicos que são à base das populações em diferentes regiões. O fenótipo pode ser influenciado por fatores ambientais e genéticos. Um entendimento dessas doenças genéticas tornase importante para a saúde e qualidade de vida da população (BONINI-DOMINGOS et al., 2004).

A molécula de hemoglobina é um tetrâmero de cadeias de globina formado por duas cadeias do tipo alfa ( $\alpha$ e $\zeta$ ) e duas do tipo não-alfa $(\beta, \gamma, \delta$ e $\xi)$, sendo a hemoglobina $\mathrm{A}$ $\left(\alpha_{2} \beta_{2}\right)$, o tipo predominante entre as hemoglobinas normais do indivíduo adulto. Conceitualmente, as hemoglobinas anormais são aquelas que apresentam estrutura diferente da hemoglobina $\mathrm{A}$, podendo também apresentar variações em relação à $\operatorname{HbA}_{2}\left(\alpha_{2} \delta_{2}\right)$ e à $\operatorname{HbF}\left(\alpha_{2} \gamma_{2}\right)$. Dados da literatura demonstram que já foram diagnosticados centenas de tipos diferentes de hemoglobinas anormais (FREIRE et al.; 2000).

As alterações nesta proteína envolvem a síntese estrutural e quantitativa dos aminoácidos que compõem as diferentes cadeias de globinas (alfa, beta, gama e delta). A gravidade da doença depende da herança transmitida, como caráter dominante, pelo (s) progenitor (es) do indivíduo. As hemoglobinopatias agrupam hemoglobinas anormais que causam hemólise, policitemia, cianose ou falcização. Algumas são mais frequentes como a $\mathrm{HbS}$ e $\mathrm{HbC}$, originadas pela substituição do ácido glutâmico, na posição número 6 da cadeia Beta, pela valina e/ou pela lisina, respectivamente (FREIRE et al., 2000).

A $\mathrm{HbS}$, quando exposta à baixa tensão de oxigênio, tende à polimerização em cristais tactóides, ocasionando uma anemia hemolítica devido à imperfeição eritrocitária. Nos indivíduos heterozigotos (HbAS), cerca de $40 \%$ da hemoglobina é do tipo $\mathrm{S}$ e aproximadamente $60 \%$ é $\mathrm{HbA}$, o que define o 
traço falciforme, com características clínicas bem mais amenas. De modo similar ao encontrado em outros países como México, Venezuela e Costa Rica, no Brasil, a HbAS é a forma mais comum. (ARENDS et al., 2007; PEÑALOSA-ESPINOSA et al., 2008; SAÉNZ-RENAULD GF, 2005).

Em indivíduos com formas heterozigóticas, verifica-se uma morbidade silenciosa, já que o indivíduo só apresenta sintomatologia clínica em raras situações críticas como hipóxia, subida a grandes altitudes, falta de $\mathrm{O}_{2}$ durante anestesias, sobrevivência a afogamentos, etc. Portanto, são eventos em que o indivíduo é submetido a baixas concentrações de oxigênio, conferindolhe a ocorrência de precipitação das moléculas de hemoglobina em forma de cristais, o que causa toda a patologia (FAILACE, 2003).

Nas formas homozigóticas, que ocorrem quando o filho herda de seus pais heterozigotos os genes recessivos responsáveis pela mutação na molécula de hemoglobina, existem inúmeras manifestações clínicas com apresentação inicial dos sintomas já nos primeiros anos de vida. Sabe-se que aproximadamente 300000 crianças nascem com anemia falciforme anualmente (a maioria na África), e outras 40 000 são muito afetadas com a $\beta$ talassemia (MODELL e DARLISO, 2008).

Neste caso, são constatadas evidentes alterações clínico-hematológicas, as quais levam a importantes limitações físicas e laborativas, a inúmeros problemas sociais e familiares, além de grandes gastos econômicos pelos serviços de saúde e uma alta taxa de morbidade e mortalidade (FREIRE et al.; 2000), decorrentes de episódios de crises vasoclusivas dolorosas, crises hemolíticas e de sequestro visceral (HOFFBRAND et al., 2006).

Com relação à $\mathrm{HbC}, \quad \mathrm{o}$ estado heterozigótico corresponde a uma condição assintomática, vivendo o indivíduo portador de maneira inteiramente normal. Geralmente, o indivíduo descobre ser portador casualmente pela eletroforese em $\mathrm{pH}$ alcalino e ácido, demonstrando a presença de duas hemoglobinas, em proporções semelhantes: HbA e HbC. Já em homozigose, a chamada doença da hemoglobina $\mathrm{C}$ tem seu quadro clínico concentrado na hemólise crônica, acompanhada de hepatoesplenomegalia (RAMALHO et al., 2003), grande quantidade de hemácias em alvo na circulação e discretos indícios de hemólise (ZAGO et al., 2004).

\section{MATERIAIS E MÉTODOS}

O estudo foi realizado com a população de 600 alunos de ambos os sexos, matriculados no ensino médio de uma escola estadual da cidade de Alfenas-MG, com idade igual ou superior a 18 anos, no período de abril a setembro de 2011 .

Todos voluntários receberam orientações quanto à sua participação na pesquisa e após assinatura do Termo de Consentimento Livre e Esclarecido (TCLE), 
procedeu-se a coleta da amostra sanguínea (3 $\mathrm{mL}$ ), realizada mediante punção venosa por profissional habilitado, utilizando-se de tubos siliconizados contendo o anticoagulante EDTA na proporção de $1 \mathrm{mg} / \mathrm{mL}$ de sangue. As amostras obtidas foram submetidas às análises laboratoriais de eletroforese de hemoglobina em pH 8,6 (alcalino) e 6,2 (ácido), bem como à prova de solubilidade. (NAOUM, 1997; VELLA, 1968 e PRUDÊNCIO, et al., 2000).

Esta pesquisa teve aprovação do Comitê de Ética em Pesquisa em Seres Humanos da Universidade Federal de Alfenas (Protocolo de Pesquisa ${ }^{\circ}$ 038/2011).

\section{RESULTADOS E DISCUSSÃO}

Com a realização do presente estudo, foi possível avaliar a ocorrência de hemoglobinopatias variantes em estudantes de Alfenas, verificando sua prevalência nesta população. Foram analisadas 600 amostras sanguíneas, sendo que $41,33 \%$, ou seja, 248 indivíduos eram do sexo masculino e que $58,67 \%$ (352 participantes), do sexo feminino.

Inicialmente, foram triadas as formas variantes de hemoglobinas por eletroforese em $\mathrm{pH}$ alcalino e, aquelas amostras que apresentaram alguma alteração no traçado eletroforético foram também analisadas mediante eletroforese em $\mathrm{pH}$ ácido para diferenciação migratória de $\mathrm{HbS}, \mathrm{HbD}, \mathrm{HbC}$ e $\mathrm{HbE}$ e confirmação da presença de hemoglobinas variantes. Com isso, pode-se obter os valores de prevalência das hemoglobinas variantes $\mathrm{HbS}$ e $\mathrm{HbC}$ na população estudada sendo observadas $96,5 \%$ indivíduos normais e 3,50\% portadores de hemoglobinas variantes.

Por fim, definiu-se a porcentagem total de indivíduos AS e AC dentre os indivíduos normais, a fim de confirmar dados de pesquisas anteriores e verificar a real proporção deste acometimento na população estudada. Este resultado pode ser observado na Figura 1.

Figura 1 - Prevalência Total de Indivíduos Portadores de $\mathrm{HbS}$ e HbC em População Estudantil de Alfenas MG.

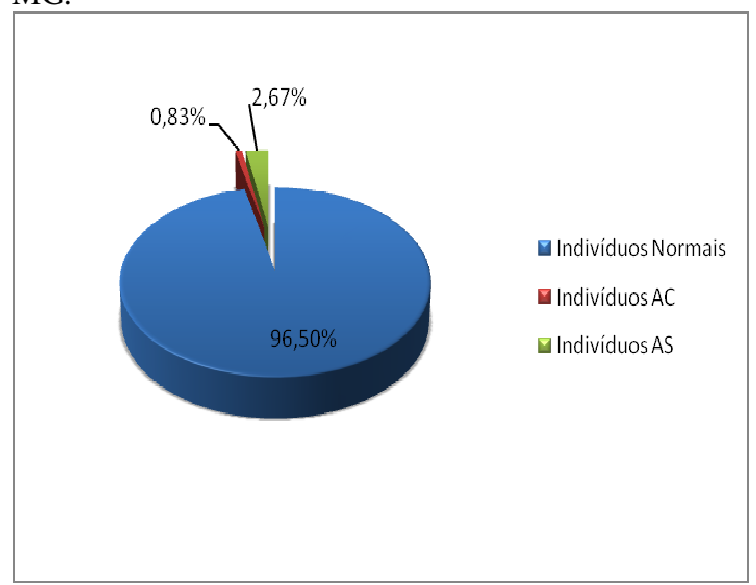

Fonte: próprio autor.

Os resultados obtidos pelas provas de solubilidade foram totalmente coerentes com o resultado preliminar da eletroforese de hemoglobina, confirmando, em seus respectivos casos, a presença de hemoglobina $\mathrm{S}$ nas amostras em questão.

Acedo et al. (2002), em um estudo com 1.846 doadores voluntários de sangue não consanguíneos, encontraram 21 portadores do traço falciforme $(1,13 \%)$ e seis do traço da 
hemoglobina C (0,32\%). Além disso, detectou-se dois portadores de Persistência Hereditária de Hemoglobina Fetal - PHHF $(0,1 \%)$, um da hemoglobina $\mathrm{J}(0,05 \%)$ e um doador portador de hemoglobina $\mathrm{D}(0,05 \%)$. No total, foram diagnosticados 31 portadores de hemoglobinopatias $(1,68 \%)$ sendo que nesta porcentagem, o traço da hemoglobina $S$ corresponde a $67,74 \%$ e o traço da hemoglobina $\mathrm{C}$ a $19,35 \%$.

Rodrigues (2010), em sua pesquisa, relata que a hemoglobina $\mathrm{S}$ foi identificada em 16 amostras sendo que oito apresentaram heterozigose para hemoglobina $(2,53 \%)$ e um, homozigose $(0,32 \%)$. Também foram identificadas quatro amostras $(1,27 \%) \mathrm{em}$ heterozigose para $\mathrm{HbC}$.

O estudo do perfil hemoglobínico, por eletroforese e prova de solubilidade, demonstrou que a população em estudo segue os padrões pré-determinados das prevalências de hemoglobinas variantes, como já comprovado por trabalhos anteriores.

Souza et al. (1998), demonstraram que em diversas regiões brasileiras, a frequência analisada das variantes oscilou de 2 a $11 \%$, confirmando a predominância das hemoglobinas $\mathrm{S}$ e $\mathrm{C}$, as quais devem ser vistas como de maior relevância no Brasil.

Obteve-se neste trabalho uma prevalência de 3,5\% de indivíduos portadores de hemoglobinas variantes, o que se encontra dentro do intervalo de frequência apresentado pelo estudo acima descrito.
De acordo com Adorno et al. (2005), foi verificado a presença de hemoglobinopatias em recém-nascidos de uma maternidade pública de Salvador-BA sendo que de 590 recém-nascidos, $101(17,4 \%)$ apresentaram variação nas hemoglobinas, dos quais 57 (9,8\%) eram heterozigóticos para $\mathrm{HbS}$, e 38 $(6,5 \%)$ foram heterozigotos para $\mathrm{HbC}$.

Além disso, verificaram que um participante $(0,2 \%)$ foi homozigótica para $\mathrm{HbS}$ e cinco $(0,9 \%)$ foram heterozigotos duplos para $\mathrm{HbS}$ e $\mathrm{HbC}$. Segundo este autor, as elevadas frequências de hemoglobina variante observada é devido à alta taxa de mistura racial na população baiana, outro ponto discutido foi que a maternidade estudada atende grande parte das mulheres de baixa renda em Salvador, que praticamente se caracteriza por negros ou mulatos. (ADORNO et al., 2005).

Para uma análise mais isolada, verificou-se que $2,67 \%$ dos indivíduos analisados foram considerados portadores de traço falcêmico, e outros $0,83 \%$ foram diagnosticados como heterozigotos para a hemoglobinopatia $\mathrm{C}$, prevalências estas que também têm sido demonstradas em outros estudos relacionados. Todas as amostras com suspeitas de presença de $\mathrm{HbS}$ foram submetidas à prova de solubilidade a fim de confirmar a presença desta variante de hemoglobina de maneira adequada.

Em um estudo com os voluntários dos programas comunitários de saúde, a frequência de portadores da $\mathrm{HbS}$ variou de 
9,5 a $11,4 \%$ e a da $\mathrm{HbC}$ ficou entre 2,3 a $2,8 \%$. A frequência total de portadores para as duas hemoglobinas nestas amostras foi de 13\%. Nesta pesquisa, o povoado de São Tiago do Iguape, uma comunidade da zona rural remanescente de quilombo no Recôncavo Baiano, foi encontrado dois pacientes falcêmicos numa amostra de 44 pessoas (SILVA et al., 2006).

Afirma-se, além disso, que nenhum indivíduo participante desta pesquisa foi diagnosticado, a partir dos exames realizados, como sendo portador homozigoto dos genes codificadores das hemoglobinas $\mathrm{S}$ e $\mathrm{C}$ e, portanto, nenhum deles apresentou resultados compatíveis com a ocorrência de anemia falciforme e/ou hemoglobinopatia C. Resultados similares foram observados em uma pesquisa com Sousa e Araújo (2005).

Por outro lado, em um trabalho envolvendo 168 municípios da região oeste do estado de São Paulo, a prevalência do traço falciforme entre recém-nascidos alcançou $2,35 \%$ e para a condição homozigota ou doença falciforme atingiu $0,004 \%$ (PULTRINI et al., 2004).

Com relação ao sexo dos indivíduos participantes, verificou-se a prevalência de $\mathrm{HbC}$ exclusivamente em mulheres, fato que não demonstra correlação com nenhum dado da literatura, podendo ser considerado decorrente da casualidade e da heterogeneidade da população estudada. Quanto à ocorrência de $\mathrm{HbS}$, verifica-se uma frequência levemente mais acentuada na população masculina comparada à população feminina estudada.

\section{CONCLUSÃO}

As hemoglobinopatias, embora tratáveis, consistem em doenças crônicas e incuráveis. Comumente causam grande sofrimento. Assim, sob os aspectos clínico, genético e psicossocial, seus portadores, mesmo que heterozigotos, devem receber atenção específica.

Sua natureza incurável ressalta a importância da prevenção. O diagnóstico precoce constitui uma vantagem, pois possibilita um direcionamento sobre o risco reprodutivo de casais heterozigóticos.

As orientações acontecem por meio de programas de aconselhamento genético as famílias positivas. Estes ocorrem através da informação aos futuros progenitores sobre a presença das características genéticas e envolvimento de possíveis riscos, de forma a colaborar na redução da incidência da doença.

Este estudo permitiu instruir a comunidade escolar por meio de atividades educativas em saúde, empregando-se materiais adequados, que possibilitaram realizar esclarecimentos individuais sobre este importante problema de saúde pública.

\section{AGRADECIMENTOS}

A Unifal-MG; ao PROEXT/MEC pelo apoio concedido. 


\section{REFERÊNCIAS}

ACEDO, M. J. et al. Programa comunitário de hemoglobinopatias: abordagem populacional a partir de doadores de sangue de Bragança Paulista, São Paulo, Brasil. Cad. Saúde Pública, Rio de Janeiro, 18(6):1799-1802, nov-dez, 2002.

ADORNO, E.V et al. Hemoglobinopathies in newborns from Salvador, Bahia, Northeast Brazil. Cad. Saúde Pública, Rio de Janeiro, 21(1):292-298, jan-fev, 2005.

ARENDS A. et al. Hemoglobinopatías em Venezuela. Interciencia. 2007;32:516-21.

BONINI-DOMINGOS, A. C. et al. Identificação de Variantes de Hemoglobina em Doadores de Sangue. Revista Brasileira de Hematologia e Hemoterapia. v.26 n.1. 2004.

FAILACE, R. Hemograma - Manual de Interpretação. $4^{\text {a }}$ Ed. Editora Artmed. 91-95. 2003.

FREIRE, N.; FERREIRA, M. C. L.; FERREIRA, S.; Prevalência de Hemoglobinopatias em Pacientes do Laboratório de Patologia Clínica da Fundação São Francisco Xavier e a sua Importância no Aconselhamento Genético. NewsLab, 116120. Ed. 43. 2000.

HOFFBRAND, A. V.; PETTIT, J. E.; MOSS, P. A. H. Fundamentos em Hematologia. $4^{\mathrm{a}}$ Ed. Editora Artmed. 65-98. 2006.

MODELL B.; DARLISO M. Global epidemiology of haemoglobin disorders and derived service indicators. Bull World Health Organ. 2008;86(6):480-487.

NAOUM, P. C.; DOMINGOS, C. R. B. Técnicas laboratoriais para identificação das hemoglobinas normais e anormais, In: NAOUM, P. C. (Ed.), Hemoglobinopatias e Talassemias. São Paulo: Sarvier. 155-156. 1997.
NAOUM, P. C. Hemoglobinopatias e Talassemias. São Paulo, Savier Ed. Livros Médicos. P. 171. 1997.

PEÑALOSA-ESPINOSA R. I. et al. Frecuencia de la hemoglobina $\mathrm{S}$ em cinco poblaciones mexicanas y su importancia en la salud pública. Salud Pública Mex. 2008;50:325-9.

PRUDÊNCIO, B. C. A. B. et al. Comparação de Metodologia Utilizada para a Detecção de Hemoglobina S ( HbS) em Doadores de Sangue. Revista Brasileira de Hematologia e Hemoterapia. 22(2), 99-109. 2000.

PULTRINIT et al. Triagem Neonatal para Hemoglobinopatias em municípios da região oeste do estado de São Paulo. Arq Ciênc Saúde. 11(1): 20-4. 2004.

RAMALHO, A. S. et al. A Portaria $n^{\circ} 822 / 01$ do Ministério da Saúde e as Peculiaridades das Hemoglobinopatias em Saúde Pública no Brasil. Caderno de Saúde Pública. 19(4): 1195-1199. 2003.

RODRIGUES, A. P. Prevalência de hemoglobinopatias e talassemias em pacientes com anemia na cidade de São Carlos. AC\&T CIENTÍfICA. vol 1. número 2. 2010.

Disponível em:

$<$ http://www.ciencianews.com.br/revistavirtua 1/arthemato.html>. Acesso em 25 fev. 2013.

SAÉNZ-RENAULD GF. Hemoglobinas anormales. Acta Med Costarric. 2005;47:173-9.

SILVA W.S. et al. Avaliação da cobertura do programa de triagem neonatal de hemoglobinopatias em populações do Recôncavo Baiano, Brasil. Cad. Saúde Pública, Rio de Janeiro, 22(12):2561-2566, dez, 2006.

SOUSA F. G. M.; ARAÚJO T. L. R. Hemoglobinopatias em população infantil de um município Maranhense. Enferm. UERJ; 13:325-330, 2005. 
SOUZA, I. P. et al. Frequência de Hemoglobinas Anormais Detectadas em Doadores de Sangue do HEMOCE.

NewsLab, Ed. 26. 96-100. 1998.

VELLA, F. Acid Agar Gel Electrophoresis of Human Hemoglobin. American Journal of Clinical Pathology. 49(3); 440-442. 1968.

ZAGO, M. A.; FALCÃO, R. P.; PASQUINI, R. Hematologia - Fundamentos e Prática. Editora Atheneu. 241-249. 2004. 